\title{
Advances in the application of respiratory training in patients with cardiovascular disease
}

\author{
Ye Yumei $^{1 *}$, Gong Beitao ${ }^{1 *}$, Hu Yaoyao ${ }^{2 \#}$
}

${ }^{1}$ Intensive Care Unit, the Seventh Affiliated Hospital, Sun Yat-sen University, Shenzhen 518107, China.E-mail: maynight99@icloud.com,https://orcid.org/0000-0002-8874-2521.

Department of Cardiovascular Disease, the Seventh Affiliated Hospital, Sun Yat-sen University, Shenzhen

518107,China; GongBeitao,E-mail:919816187@qq.com.https://orcid.org/0000-0002-5286-2468.

${ }^{2}$ School of Nursing, Soochow University, Suzhou 215006, China,E-mail:2464343847@qq.com, https://orcid.org/00000002-9088-6516.

* Ye Yumei and Gong Beitao are co-first authors. \#Hu Yaoyao is the corresponding author.

\section{Article History Received 28 March 2020 Accepted 29 April 2020 Published 30 June 2020}

Cite this Article Ye Yumei, Gong Beitao, Hu Yaoyao. Advances in the application of respiratory training in patients with cardiovascular disease [J].Medical Research, 2020.2(2):9-15, http://dx.doi.org/10.6913/MRHK. 202006_2(2).0002

Copyright C 2020 Creative Publishing Co., Limited. All rights reserved. mrhk26640333@gmail.com.

\begin{abstract}
As one of the lifestyle interventions, respiratory training has a positive role in rehabilitation. This study summarizes the mechanism, intervention technique, effects and problems of respiratory training in patients with cardiovascular disease, so as to provide reference for the developing of the best respiratory training program of the clinicians.
\end{abstract}

Key words Cardiovascular Disease; Respiratory Rehabilitation Training; Review

Cardiovascular disease is a composite of a group of diseases, including CHD, stroke, hypertension, etc., caused by multiple factors. It is the leading cause of death in the world ${ }^{[1]}$, and also the leading cause of death in China ${ }^{[2-}$ ${ }^{3]}$. At present, the main treatments for cardiovascular disease are drug treatment and surgical treatment and so on, while surgery can not change the pathological progression of the disease and long-term drug use will bring side effects. Therefore, it is very necessary to find a new method for the therapy of cardiovasular disease. According to some studies at home and overseas, respiratory training did lower the resting heart beat and blood pressure of the patients with cardiovascular disease against the characteristics of cardiovascular disease, helping with the prognosis of the patients ${ }^{[4-7]}$. Thus it is an effective adjuvant therapy and one of the therapies for the patients overseas as a new economical technology of rehabilitation ${ }^{[8]}$. Therefore, this paper aimed to summary the relevant researches on respiratory training in patients with cardiovascular disease and provide reference for the future breathing training carried out in clinical trials.

\section{Overview of Respiratory Training}

\subsection{Definition}


Ye Yumei et al. Advances in the Application of Respiratory Training in Patients with Cardiovascular Disease Medical Research ISSN 2664-0333 eISSN 2664-0341 Volume 2 Issue 2 http://dx.doi.org/10.6913/MRHK.202006_2(2).0002

Respiratory Rehabilitation Training, also known as pranayam and respiratory feedback, is a training method for subjects in which certain breathing patterns are invloved, to achieve a balance of physiological functions ${ }^{[9]}$.

\subsection{Mechanism}

Some studies showed that pathological changes in cardiovascular disease may lead to cardiac autonomic nervous system dysfunction ${ }^{[10,11]}$, aggravating the progression of arterial sclerosis, which makes arterial elasticity decrease, further causing increased blood pressure, increasing consumption of myocardial oxygen. In severe cases, even malignant arrhythmia would be caused, endangering patients' life $^{[12-13]}$. Besides, the onset and development of the disease will also make patients prone to anxiety, depression and other adverse mental problems ${ }^{[14]}$, and the loss of the quality of life ${ }^{[15]}$ and sleep ${ }^{[16]}$. However, respiratory training can maintain or reconstruct the balance of autonomic nerve functions, stabilizing the patient's heart rate, blood pressure ${ }^{[17]}$, and improving cardiovascular functions ${ }^{[18]}$. Respiratory training was used in patients with respiratory-related diseases at first and some patients who received a thoracic surgery, proving its safety and efficacy ${ }^{[19-21]}$. The statements above provided a practical and theoretical basis for respiratory training working as a adjuvant treatment of cardiovascular diseases.

\section{Application of respiratory training in patients with CVD}

\subsection{Respiratory training techniques}

At present, the respiratory intervention techniques applied to CVD patients, such as abdominal respiration, respiratory training of inspiratory muscles achieved good results.

\subsubsection{Abdominal respiration}

Soley ${ }^{[22]}$ first proposed Abdominal respiration method, which requires the subjects to breath evenly, deeply and thinly. It is a regular breath method of strengthening the movement of diaphram. The increasing of the range of the motion and the quantity of the gas exchange of the lungs by the movement of diaphram will increase the abdominal pressure and boost the blood circulation of the abdomen, stablizing and balancing the automatic nervous functions ${ }^{[23]}$. Some scholars believe that the effect of the reduction of blood pressure would be the best by using abdominal respiration at a frenquency of 8 times per minute ${ }^{[24]}$, or 10 times per minute ${ }^{[25]}$ left disunified yet. However, such a low-frequency breathing is too difficult for patients having received the training for the first time to accept. Other scholars propose a method of guiding the respiration training with the help of a breath feedback machine. They collect the data of the patients' abdominal respiratory waves and work out the current respiration rate. Thus the machine generates musical rhythms or melodies accordingly to guide the patients' breathing, greatly lowering the patients' blood pressure ${ }^{[26]}$. But this method needs to be done with the machine, it would be difficult for discharged patients to be trained this way again.

\subsubsection{Inspiratory muscle training}

\section{(1) resistance training}

Resistance training is a training method that patients exercise their muscles with the inhalation capacity by overcoming certain degree of resistance, so as to improve the muscle strength and durability and further strengthening patients'cardio-pulmonary functions ${ }^{[27-28]}$. Inspiratory muscle training is to intervene with specific machines, such as nonlinear resistance respirators, threshold pressure load trainers, etc. Valkenet $\mathrm{K}^{[29]}$ et al. gave preoperative resistance inspiratory muscle training to patients who were going be receive coronary bypass, reducing lung complications after surgery and the duration of hospital stay. Whilst such training requires the quantitative guidance of the therapist as well as rather expensive machines, to popularize it clinically would be challenging.

\section{(2) Fast and slow breathing}


Ye Yumei et al. Advances in the Application of Respiratory Training in Patients with Cardiovascular Disease Medical Research ISSN 2664-0333 eISSN 2664-0341 Volume 2 Issue 2 http://dx.doi.org/10.6913/MRHK.202006_2(2).0002

Fast and slow breathing is a method to inhale fast and exhale slowly ${ }^{[30]}$. According to a study of Gu Jun ${ }^{[31]}$, the exercise tolerance of the inpatients with chronic heart is improved after they undergo the fast and slow breathing training for three times a day, 10 minutes per time, 6 times per minute. However, the training compliance of the patients can't be guaranteed given a rather low frequancy of breath and the lack of the supversion and guidance of medcial staff after hospiral discharge.

\subsection{Frequency, cycle and timing of respiratory training}

Studies show there will be improvement of the functions of respiratory muscles after at least 5 weeks of the respiratory training ${ }^{[32]}$. The effect respiratory training have on patients' physiological status. Therefore, according several studies $\left.{ }^{[31,33,34}\right]$, patients are required to do the training for 4 to 12 weeks, three times per day at least, 10 to 15 minutes each time. While according to his systematic review, Martín ${ }^{[35]}$ suggests that patients should do the training once a day, 15 to 30 minutes each time. Respiratory training will put more pressure on patients' abdominal. That's why some studies ${ }^{[30]}$ suggested that 1 or 2 hours after meals is the best time for patients to do the training. Another study ${ }^{[33]}$ suggested that the last training during a day should be conducted before bedtime. Despite the diversity of the training techniques mentioned above, all of them have affirmed the value of respiratory training.

\subsection{Effect of respiratory training in patients with CVD}

\subsubsection{Effect on heart rate of CVD patients}

As a vital sign that can be acquired most easily, heart rate reflects the health status of the body and is an important factor that will affect the occurrence and development of various cardiovascular diseases, such as hypertension, acute myocardial infarction and so on $^{[36-38]}$. After conducting respiratory interventions on subjects for 3 months (ratio of exhalation to inhalation time 2:1), Adhana $\mathrm{R}^{[39]}$ et al. found that the heart rates of the subjects in the intervention group was lowered. SHI Xunyu ${ }^{[34]}$ et al. performed rapid and slow respiration intervention on patients with chronic heart failure, lowing their resting heart rates. WU Qing ${ }^{[7]}$ et al. proved that respiratory training is helpful in improving the status of the heart rate of patients with CVD after conducting 12 weeks of spontaneous respiratory training on patients with stable coronary heart disease. The machanism of the effect is likely to be respiratory movement changing cardiac output and intrathoracic pressure, which stimulate the cardiopulmonary receptors, further inhibiting the excitement of central nervous system and finally reducing the heart rate. Therefore, for patients with CVD, respiratory training can help improve the status of heart rate of the patients with CVD and the result of their prognosis.

\subsubsection{Effects on blood pressure of CVD patients}

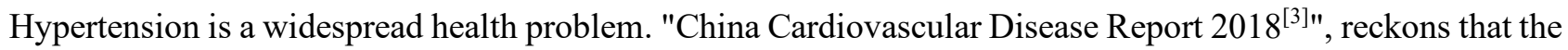
total number of patients with hypertension in China is 245 million. Hypertension is one of the main risk factors for deaths of cardiovascular diseases in China. According to statistics, hypertention is the cause of nearly half of the deaths of patients with cardiovascular diseases ${ }^{[40]}$. One study has shown that lower blood pressure may help reduce the incidence of cardiovascular diseases in patients with CVD. Several studies proved that doing deep and slow respiratory training can help patients with primary hypertension lower their blood pressure ${ }^{[24]}$. According to a study on sutomatic respiratory training interventions in patients with coronary heart disease, breathing training can lower blood pressure in patients with coronary heart disease ${ }^{[7]}$. Therefore, respiration intervention in patients with CVD helps improve their condition of blood pressure.

\subsubsection{Effect on heart rate variability in CVD patients}

Heart rate variability refers to nuances between cardiac cycles. It analyzes the subtle difference of duration and pattern of each cardiac cycle. It is one of the indicators in quantitative assessment of automatic nervous 
Ye Yumei et al. Advances in the Application of Respiratory Training in Patients with Cardiovascular Disease Medical Research ISSN 2664-0333 eISSN 2664-0341 Volume 2 Issue 2 http://dx.doi.org/10.6913/MRHK.202006_2(2).0002

functions ${ }^{[42-43]}$ and one of the predictors of cardiovasular diseases ${ }^{[44]}$. Heart rate variability is mainly tested by 24-hour time domain analysis indicators and calculated and analyzed automatically by computer. Several studies ${ }^{[7,17,24]}$ showed that respiratory training can increase the time domain index of heart rate variability in patients with primary hypertension or coronary heart disease, possibly because breathing movement stabilizes the autonomic nerve functions, that is to say, reduce sympathetic nerve activities while increase parasympathetic nerve activities. Therefore, it is necessary for patients with CVD to carry out respiratory training to stabilize or reconstruct their automatic nerve functions.

\subsubsection{Effect on living quality, sleep quality, anxiety and depression in CVD patients}

CVD shall have certain influence on patients' work, daily life and so on. Therefore, the quality of life is more and more often used to assess the effects of respiratory training. Several studies ${ }^{[29,33,34]}$ have shown that respiratory training can improve patients' living quality and sleep quality and relieve negative psychologies, such as anxiety and depression. On one hand, it may be because breathing training improves patient's heart and lung functions, strengthening their self-care abilities in daily life, finally improving their living quality. On the other hand, it may be because that the improvement in physiological status, namely heart and lung functions convince that their diseases are relieved, and thus their negative emotions are relieved.

\section{The research status}

Respiratory training is an important complementary treatment for patients with CVD. At present, however, most of the researches are on the respiratory training in patients with cardiovascular diseases like hypertension, chronic cardiac failure and stroke, while that in patients with coronary heart disease is relatively few, especially in China.

\subsection{Lack of unified operation specification}

Respiratory training is rather new in China. Clinial staff don't have adequate guidelines or consensus for reference in practice. Besides, the operation of respiratory training varies given different medical envrionments, cultural environment, human resources and so on. Therefore, there is a lack of standard and unified management plan of respiratory training. Considering the problems above, the practices of overseas respiratory training groups shall be used as a reference to help with the construction of our own nursing intervention plan.

\subsection{Lack of evidence-based support}

In China, respiratory training for patients with CVD most of the time is guided by nursing staff, most on health education and guidance, in the aim of improving patients' compliance and standardization of the training. The present nursing intervention programs which is constructed by relevant research institutes mostly based on their own nursing experience lacks of systematical analysis and integrate methods on how to assess the right patients to do the training, how to choose the right option of the training and how often should the followup visit be. Therefore, the nursing program lacks of evidence-based support. Clinical staff could analyze and integrate such studies so as to construct our own respiratory training program suitable for CVD patients to improve their prognosis.

\subsection{Lack of continuity}

The popularization of inspiratory muscle training in clinical practice is quite hard for the expensive apparatuses requested and assistance from therapists. Patients have no access to them after discharge, therefore they have to quit the training. Besides, a long time of the training might weaken patients' compliance and make them quit the training. Therefore, nursing staff should construct a practical respiratory training program suitable for CVD patients in China, in order to ensure the continuity of patients after discharge. 
Ye Yumei et al. Advances in the Application of Respiratory Training in Patients with Cardiovascular Disease Medical Research ISSN 2664-0333 eISSN 2664-0341 Volume 2 Issue 2 http://dx.doi.org/10.6913/MRHK.202006_2(2).0002

\section{Summary}

The positive effects of respiratory training on CVD patients has been recognized. It works as an important complementary treatment for them. However, there are relatively few researches on the effects on it in patients with coronary heart diseases. It is suggested that more searches on it should be conducted. In addition, inspiratory muscle training requires necessary equipments and apparatuses and therapists' assistance. Patients can not continue the training after discharge for a lack of access to expensive equipments and assistance from therapists, while their physiological indexes shall be improved only after a long time of the training. The effects of inspiratory training in some patients are not good enough because they can not continue the training for long. Therefore, in the future, there should be more intensive searches on the respiratory training for CVD patients. Meanwhile, the training program should be finalized and unified for a wider use in clinical practice. Besides, complicance of the patients should also be taken into account.

\section{STATEMENT}

There is no conflict of interest in this article.

\section{REFERENCES}

1 Naghavi, M., et al.Global, regional, and national age-sex specific mortality for 264 causes of death,1980-2016: a systematic analysis for the Global Burden of Disease Study 2016[J].The Lancet, 2017,390(10100): 1151-1210.

2 Li Weizheng, Liu Shixuan, Zeng Xin, et al.1990-2016 Report on the Burden of Cardiovascular Diseasein disease in China and provincial administrative regions[J].China Circulation Journal, 2019,34 (08): 729-740.

$3 \mathrm{Hu}$ shengshou, Gao Runlin, Liu lisheng, et al. Summary of cardiovascular disease report 2018 in China [J]. Chinese journal of circulation,2019,34(03):209-220.

4 Ge Junbo, Xu Yongiian et al. Internal Medicine [M].8th edition. Beijing: People's Medical Publishing House,2013:234235.

5 Dixhoorn J. Cardiorespiratory effects of breathing and relaxation instruction in myocardial infarction patients[J]. Biological psychology. 1998: 123-35.

6 Westerdahl E,Olsén M F.Chest physiotherapy and breathing exercises for cardiac surgery patients in Sweden-A National survey of practice[J].Monaldi Archives for Chest Disease,2015,75(2):112-119.

7 Wu Qing,Wang Xiaohua,Huang Hui,et al. Effects of spontaneous breathing exercise on heart rate variability and myocardial oxygen consumption in patients with stable coronary heart disease [J]. Chinese journal of gerology,2019,39(05):1034-1037.

8 Prasad K,Sharma V,Lackore K,et al. Use of complementary therapies in patients with cardiovascular disease[J].American journal of cardiology,2013,111(3):339-345.

9 Wang Miao,Liu huaxia,Wan Xueying,et al. Application status and enlightenment of respiratory training in nonrespiratory diseases [J]. Chinese journal of nursing,2013,48(11):1030-1032.

10 Gao Lihua. Relationship between blood pressure rhythm and autonomic nervous function in well-controlled hypertensive patients [D]. Chongqing Medical University,2017.

11 Seki A,Green HR,Chen PS, et al.Sympathetic nerve fibers in human cervical and thoracic vagus nerves[J].Heart Rhythm,2014,11(8):1411-1417.

12 Thayer JF,Lane RD.The role of vagal function in the risk for cardiovascular disease and mortality[J].Biological Psychology,2007,74(2):224-242.

13 Lin IM,ai LY,Fan SY.Breathing at a rate of 5.5 breaths per minute with equal inhalation-to-exhalation ratio increases heart rate variability[J].International Journal of Psychophysiology,2014;91:206-211.

14 Minhua,Li Jingzhi,Chen Ling,et al.Coronary heart disease patients with negative emotions 132 cases retrospective 
Ye Yumei et al. Advances in the Application of Respiratory Training in Patients with Cardiovascular Disease

Medical Research ISSN 2664-0333 eISSN 2664-0341 Volume 2 Issue 2 http://dx.doi.org/10.6913/MRHK.202006_2(2).0002

analysis[J].Guangdong Medicine,2019,40(19):2748-2753.

15 Zhang Yu, Lan Jian.Coronary heart disease combined with the healthy quality of life of patients with heart failure related to potential biomarker[J].Chinese Journal of Cardiovascular Sciences,2019,24(6):541-544.

16 Zhang Ruili, Zhang Yuxiao, Zhang Xinping,et al.Home sleep quality and sleep beliefs and attitudes in patients with stable coronary heart disease [J]. Chinese journal of practical nursing,2019,35(24):1859-1864.

17 Mohamed L A, Hanafy N F, Elnaby A G, et al. Effect of slow deep breathing exercise on blood pressure and heart rate among newly diagnosed patients with essential hypertension[J]. Journal of Education and Practice, 2014, 5(4): 36-45.

$18 \mathrm{Pal} \mathrm{G}$, Agarwal A, Karthik S, et al. Slow yogic breathing through right and left nostril influences sympathovagal balance, heart rate variability, and cardiovascular risks in young adults [J]. North American Journal of Medical Sciences, 2014, 6(3): 145-151.

19 Liu jia.Effect of intermittent breathing training on negative mood and treatment compliance in elderly patients with chronic obstructive pulmonary disease [J].International journal of geriatrics,2019,40(2):104-106.

10 Du Suzhi. Effects of Load Breathing Training on Lung Function and Quality of Life in Pneumoconiosis Patients[J].Chinese Journal of Nursing,2010,45(3):237-239.

21 Zou Yuchan,Wu Zhaohong,Chen Xingqing,et al. Effect of acupuncture combined with respiratory training on respiratory function of patients after tracheotomy after stroke [J]. Chinese journal of rehabilitation medicine,2019,34(12):1470-1473.

22 Soley MD, Shock NW.The etiology of effort syndrome[J].Am J Med-Sci, 1938, 196:840-851.

23 Tang Mingjing.Effects of breathing relaxation training on physiological response and fatigue [D]. Jiangsu: Soochow University,2018.

24 Chang Q H, Liu R H, Li C J, et al. Effects of slow breathing rate on blood pressure and heart rate variabilities in essential hypertension[J]. Int J Cardiol, 2015, 185(1): 52-54.

25 Landman G W, van Hateren K J, van Dijk P R, et al. Efficacy of device-guided breathing for hypertension in blinded, randomized, active-controlled trials: a meta-analysis of individual patient data[J]. JAMA Intern Med, 2014, 174(11): 1815-1821.

26 Diao Z J, Liu H Y, Zhu L, et al. Therapeutic hypertension system based on a microbreathing pressure sensor system[J]. Med Devices (Auck1), 2011, 4: 51-57.

27 Tian Jiawei, Cai Liyuan, Hou Wei. Respiratory trainerins in the stable period of chronic obstructive pulmonary disease patients in the current status of the application of the lung rehabilitation[J].China Rehabilitation Theory and Practice,2018,24 (4): 416-421.

28 Cho JE, Lee HJ, Kim MK, et al. The improvement in respiratory function by inspiratory muscle training is due to structural muscle changes in patients with stroke: a randomized controlled pilot trial[J].Top Stroke Rehabil, 2018, 25 (1) :37-43.

29 Valkenet K, Trappenburg JCA, Hulzebos EH, et al. Effects of a pre-operative home-based inspiratory muscle training programme on perceived health-related quality of life in patients undergoing coronary artery bypass graft surgery[J]. Physiotherapy, 2017,103(3):276-282.

30 Shi Xunyu, Xu Jingjuan, Wu Wenjun, et al. Effect of fast inhalation and slow exhalation training on the improvement of exercise endurance in patients with chronic heart failure [J]. China journal of nursing,2016,51(10):1161-1165.

$31 \mathrm{Gu}$ Jun. Effect of model education combined with fast and slow breathing training on exercise endurance and functional exercise compliance of patients with chronic heart failure [J]. Nursing practice and research,2019,16(16):54-56.

32 Ramirez-Sarmiento A, Orozco-Levi M, Guell R, et al. Inspiratory muscle training in patients with chronic obstructive pulmonary disease: structural adaptation and physiologic outcomes [J].Am J Respir Crit Care Med, 2002, 166 (11) :1491-1497.

33 Wang Yan, Li Wei. Emotional Care Joint Lip-Abdominal Respiratory Training on the Impact of Difficult Chronic Heart Failure With Sleep Disorders In Pittsburgh Sleep Quality Index, Adverse Emotions, Heart Function and Quality of 
Life[J].Modern Chinese and Western Medicine Joint Journal, 2019,28 (34): 3858-3862.

34 Yao Xianli,Wu Zhiyuan,li Kunbin,et al. Effects of isokinetic muscle strength training combined with anti-obstructive respiratory training on motor function and daily life ability of patients after stroke [J]. Guangdong med medicine,2019,40(24):3383-3387.

35 Martín-Valero R, De La Casa Almeida M, Casuso-Holgado MJ,et al. Systematic Review of Inspiratory Muscle Training After Cerebrovascular Accident[J] Respir Care.,2015,60(11):1652-1659.

36 Cook S, Hess O M. Resting heart rate and cardiovascular events: time for a new crusade?[J]. European heart journal, 2010, 31(5): 517-519.

37 Palatini P, Dorigatti F, Zaetta V, et al. Heart rate as a predictor of development of sustained hypertension in subjects screened for stage 1 hypertension: the HARVEST Study[J]. Journal of hypertension, 2006, 24(9): 1873-1880.

38 Zheng Hongjian,Lu Xinzheng.Advances in cardiovascular pathophysiology with increased heart rate [J].Advances in cardiovascular epidemiology,2011,32(05):699-702.

39 Adhana R, Gupta R, Dvivedii J, et al. The influence of the 2: 1 yogic breathing technique on essential hypertension[J].Indian J Physiol Pharmacol,2013,57(1): 38-44.

40 Lewington S, Lacey B, Clarke R, et al.The burden of hypertension and associated risk for cardiovascular mortality in China[J].JAMA Intern Med, 2016, 176 (4) :524-532.

$41 \mathrm{Gu}$ D, He J, Coxson PG, et al.The cost-effectiveness of low-cost essential antihypertensive medicines for hypertension control in China:a modelling study[J].PLoS Med, 2015, 12 (8) :e1001860.

42 Feng Jun,Luo Chunmiao,Zhang Jing,et al. Influence of heart rate variability time-domain indexes on coronary artery stenosis in patients with stable angina [J]. Anhui medical science,2016,37(12):1480-1483.

$43 \mathrm{Wu}$ YK, Huang CY,Yang MC,et al. Effect of tiotropium on heart rate variability in stable chronic obstructive pulmonary disease patients[J].Journal of Aerosol Medicine \& Pulmonary Drug Delivery,2014,28(2):100-105.

44 Publishing S.Heart Rate Variability, Standard of Measurement, Physiological Interpretation and Clinical Use in Mountain Marathon Runners during Sleep and after Acclimatization at $3480 \mathrm{~m}$. World Latest Medicine Information,2013,03(1):2. 